\title{
A type of non-equivalent pseudogroups. Application to foliations
}

\author{
by Jesús A. Alvarez López (Santiago de Compostela)
}

\begin{abstract}
A topological result for non-Hausdorff spaces is proved and used to obtain a non-equivalence theorem for pseudogroups of local transformations. This theorem is applied to the holonomy pseudogroup of foliations.
\end{abstract}

Introduction. The holonomy pseudogroups of a foliation $\mathcal{F}$ are very important examples of pseudogroups since they contain the whole information about the transverse geometric structure of $\mathcal{F}$.

The definition of an equivalence of pseudogroups is given in [H2]. This notion is defined in such a way that all the holonomy pseudogroups of a foliation are equivalent. Then, using any representative of the holonomy pseudogroup one can define important invariants of the foliation [H1], [H2].

In this paper we prove a theorem giving some sufficient conditions for two pseudogroups to be non-equivalent (Section 2). In the proof of this result we use a lemma about non-Hausdorff spaces. The proof of this lemma is the most difficult part of this work (Section 3), and examples are given to clarify its hypotheses (Section 4).

Finally, the above theorem is applied to the holonomy pseudogroups of a type of foliations (Section 5), yielding properties of the space of leaves of the liftings of those foliations to certain covering spaces.

The author would like to thank the referee for helpful remarks.

1. Equivalences of pseudogroups. A pseudogroup $\mathcal{H}$ of local transformations of a topological space $T$ is a collection of homeomorphisms of open sets of $T$ such that:

(i) The composition of elements of $\mathcal{H}$, whenever it is defined, is in $\mathcal{H}$, and so are the identity map of $T$ and the inverse of any element of $\mathcal{H}$.

1991 Mathematics Subject Classification: Primary 57R30.

Key words and phrases: pseudogroup, foliation, holonomy. 
(ii) The restriction of any element of $\mathcal{H}$ to an open set is in $\mathcal{H}$.

(iii) If $h: U \rightarrow V$ is a homeomorphism of open subsets of $T$ and if there is an open covering $\mathcal{U}$ of $U$ such that the restriction of $h$ to any element of $\mathcal{U}$ is in $\mathcal{H}$, then $h$ is in $\mathcal{H}$.

Let $\mathcal{H}$ and $\mathcal{H}^{\prime}$ be pseudogroups of local transformations of spaces $T$ and $T^{\prime}$ respectively. An equivalence $\Phi: \mathcal{H} \rightarrow \mathcal{H}^{\prime}$ is defined in [H2] as a collection of homeomorphisms of open subsets of $T$ onto open subsets of $T^{\prime}$ such that $\Phi=\mathcal{H}^{\prime} \Phi \mathcal{H}$, and so that $\mathcal{H}$ (respectively $\mathcal{H}^{\prime}$ ) is generated by $\Phi^{-1} \Phi$ (respectively $\Phi \Phi^{-1}$ ). So the domains (respectively images) of the elements of $\Phi$ form a covering of $T$ (respectively $T^{\prime}$ ).

An equivalence of pseudogroups induces an equivalence of the associated topological groupoids in the sense of [H1].

We will say that a pseudogroup $\mathcal{H}$ of local transformations of a space $T$ is quasi-analytical if any homeomorphism of $\mathcal{H}$ defined on a connected open set $U$ is the identity whenever its restriction to some non-empty open subset of $U$ is the identity. We will say that $\mathcal{H}$ is complete if for any $x, y \in T$ there are open neighborhoods, $V_{x}$ of $x$ and $V_{y}$ of $y$, such that any germ of a transformation of $\mathcal{H}$ with source in $V_{x}$ and target in $V_{y}$ is the germ of an element of $\mathcal{H}$ defined on the whole of $V_{x}$.

The referee has pointed out that the property of being complete is not preserved under equivalences of pseudogroups. For example, let $\mathcal{H}$ be the pseudogroup on $\mathbb{R}$ generated by a homotethy $x \mapsto \lambda x$, with $0<|\lambda|<1$. Then $\mathcal{H}$ is complete and equivalent to its restriction to $(-1,1)$ which is not complete. Nevertheless, completeness is invariant under equivalences for pseudogroups of local isometries [H3].

The property of being quasi-analytical is not preserved by equivalences either. However, if $\mathcal{H}$ is a quasi-analytical pseudogroup equivalent to a pseudogroup $\mathcal{H}^{\prime}$ of local transformations of a Hausdorff space, then $\mathcal{H}^{\prime}$ is quasi-analytical.

A group $G$ of homeomorphisms of a topological space will be said to be quasi-analytical if the pseudogroup generated by $G$ is quasi-analytical. We have the following result with easy proof.

(1.1) Lemma. Let $G_{1}$ and $G_{2}$ be groups of homeomorphisms of spaces $T_{1}$ and $T_{2}$ respectively, $h: G_{1} \rightarrow G_{2}$ an injective homomorphism, and $f: T_{1} \rightarrow T_{2}$ an open mapping which is h-equivariant $(f g=h(g) f$ for all $\left.g \in G_{1}\right)$. Then, if $G_{2}$ is quasi-analytical so is $G_{1}$.

2. A type of non-equivalent pseudogroups. Let $T$ be a topological space. For each $x \in T$ we shall denote by $S(x)$ the subspace of points $y \in T$ such that $y \neq x$ and each neighborhood of $x$ intersects each neighborhood of $y$. 
Let us consider the following properties that $T$ may satisfy:

(A) There exists some $x \in T$ such that $S(x)$ has some isolated point.

(B) There exist $x_{1}, x_{2} \in T$ such that $x_{2} \in S\left(x_{1}\right)$ and there is a local neighborhood base $\beta_{i}$ of each $x_{i}(i=1,2)$ so that $V_{1} \cap V_{2}$ has a finite number of connected components for all $V_{1} \in \beta_{1}$ and $V_{2} \in \beta_{2}$.

A mapping $f: T \rightarrow T^{\prime}$ between topological spaces will be called locally injective if each $x \in T$ has a neighborhood $V_{x}$ such that the restriction of $f$ to $V_{x}$ is injective. Then the following lemma can be easily proved.

(2.1) Lemma. If $f: T \rightarrow T^{\prime}$ is a locally injective continuous mapping and $T^{\prime}$ is Hausdorff, then either $S(x)=\emptyset$ or $S(x)$ is discrete for all $x \in T$; thus $T$ is either Hausdorff or satisfies (A).

The following result will be proved in the next section.

(2.2) Main Lemma. Let $T$ be a locally connected topological space satisfying one of the following properties:

(i) T satisfies (A) and each point of $T$ has some compact Hausdorff neighborhood.

(ii) $T$ satisfies $(\mathrm{B})$.

Then there exist $x_{1}, x_{2} \in T$ such that for all neighborhoods $U_{1}$ of $x_{1}$ and $U_{2}$ of $x_{2}$, there exist $y_{1}, y_{2} \in T$ and there exists a connected open subset $P \subset U_{1} \cap U_{2}$ so that $y_{1} \in U_{1}, y_{2} \in U_{2} \cap S\left(y_{1}\right)$, and $P \cap Q_{1} \cap Q_{2} \neq \emptyset$ for all neighborhoods $Q_{1}$ of $y_{1}$ and $Q_{2}$ of $y_{2}$.

In Section 4 we shall see an example showing the necessity of the assumptions of $(2.2)$.

(2.3) TheOREM. Let $\mathcal{H}$ and $\mathcal{H}^{\prime}$ be pseudogroups of local transformations of spaces $T$ and $T^{\prime}$ respectively. If $T$ satisfies the hypotheses of (2.2), $T^{\prime}$ is Hausdorff, $\mathcal{H}$ is quasi-analytical and $\mathcal{H}^{\prime}$ is complete, then $\mathcal{H}$ and $\mathcal{H}^{\prime}$ are not equivalent.

P r o of. Suppose that there exists an equivalence $\Phi: \mathcal{H} \rightarrow \mathcal{H}^{\prime}$ and take $x_{1}, x_{2} \in T$ as in (2.2). Then each $x_{i}$ has an open neighborhood $U_{i}$ with a homeomorphism $\varphi_{i}: U_{i} \rightarrow V_{i}$ in $\Phi(i=1,2)$. By (2.2) there exist $y_{1}, y_{2} \in T$ and there exists a connected open subset $P \subset U_{1} \cap U_{2}$ such that $y_{1} \in U_{1}$, $y_{2} \in U_{2} \cap S\left(y_{1}\right)$, and $P \cap Q_{1} \cap Q_{2} \neq \emptyset$ for all neighborhoods $Q_{1}$ of $y_{1}$ and $Q_{2}$ of $y_{2}$.

The mapping $\varphi_{2} \varphi_{1}^{-1}: \varphi_{1}(P) \rightarrow \varphi_{2}(P)$ is in $\mathcal{H}^{\prime}$. Since $\mathcal{H}^{\prime}$ is quasianalytical (because so is $\mathcal{H}$, and $T^{\prime}$ is Hausdorff) and complete, there exists a neighborhood $W_{i} \subset V_{i}$ of each $\varphi_{i}\left(y_{i}\right)(i=1,2)$ and there exists a homeomorphism $h^{\prime}: W_{1} \rightarrow W_{2}$ in $\mathcal{H}^{\prime}$ such that

$$
h_{\mid W_{1} \cap \varphi_{1}(P)}^{\prime}=\varphi_{2} \varphi_{1}^{-1} \mid W_{1} \cap \varphi_{1}(P) \cdot
$$


Then $h=\varphi_{2}^{-1} h^{\prime} \varphi_{1}: \varphi_{1}^{-1}\left(W_{1}\right) \rightarrow \varphi_{2}^{-1}\left(W_{1}\right)$ is in $\mathcal{H}$.

Since $T^{\prime}$ is Hausdorff it is easy to check that $h^{\prime} \varphi_{1}\left(y_{1}\right)=\varphi_{2}\left(y_{2}\right)$, so $h\left(y_{1}\right)=y_{2}$. Therefore, because $\mathcal{H}$ is quasi-analytical and the restriction of $h$ to $\varphi_{1}^{-1}\left(W_{1}\right) \cap P$ is the identity, we obtain $y_{1}=y_{2}$, which is a contradiction.

3. Proof of the Main Lemma. Let $T$ be a locally connected topological space satisfying (i) of (2.2). Then there exists $x_{1} \in T$ with an isolated point $x_{2}$ in the space $S\left(x_{1}\right)$. Let $U_{1}$ and $U_{2}$ be open neighborhoods of $x_{1}$ and $x_{2}$ respectively. The connected components of $U_{1} \cap U_{2}$ are open because $T$ is locally connected. Moreover, we can assume that $U_{1}$ and $U_{2}$ are Hausdorff and locally compact, and $U_{2} \cap S\left(x_{1}\right)=\left\{x_{2}\right\}$. Then we obtain the following properties (the first two with very easy proofs).

(3.1) If $Q_{1}$ and $Q_{2}$ are neighborhoods of $x_{1}$ and $x_{2}$ respectively, then

$$
x_{i} \in \mathrm{Cl}_{U_{i}}\left(Q_{1} \cap Q_{2} \cap U_{i}\right) \quad \text { for } i=1,2 .
$$

(3.2) For each connected component $W$ of $U_{1} \cap U_{2}$ we have

$$
\partial_{U_{1}}(W) \cap \partial_{U_{2}}(W)=\emptyset .
$$

(3.3) Let $W$ be a connected component of $U_{1} \cap U_{2}$. If each $z \in \partial_{U_{2}}(W)$ has an open neighborhood $V_{z} \subset U_{2}$ such that $\mathrm{Cl}_{U_{1}}\left(V_{z} \cap W\right) \cap \partial_{U_{1}}(W)=\emptyset$, then for each neighborhood $Q$ of $x_{2}$ in $U_{2}$ with $\mathrm{Cl}_{U_{2}}(Q)$ compact we have

$$
\mathrm{Cl}_{U_{1}}(Q \cap W) \cap \partial_{U_{1}}(W)=\emptyset .
$$

Proof. We can suppose that $Q \cap W \neq \emptyset . \partial_{U_{2}}(W) \cap \mathrm{Cl}_{U_{2}}(Q)$ is a compact subspace contained in the union of the open sets $V_{z}$ (for $z \in \partial_{U_{2}}(W)$ ), so for a finite number of points $z_{1}, \ldots, z_{r} \in \partial_{U_{2}}(W)$ we have

$$
\partial_{U_{2}}(W) \cap \mathrm{Cl}_{U_{2}}(Q) \subset V_{z_{1}} \cup \ldots \cup V_{z_{r}} \quad(=V) .
$$

Then we obtain

$$
\left(\mathrm{Cl}_{U_{2}}(Q) \cap W\right)-V=\left(\mathrm{Cl}_{U_{2}}(Q) \cap \mathrm{Cl}_{U_{2}}(W)\right)-V,
$$

which is closed in $U_{2}$ and contained in $\mathrm{Cl}_{U_{2}}(Q)$. Thus $\left(\mathrm{Cl}_{U_{2}}(Q) \cap W\right)-V$ is a compact subset of $U_{1}$, so it is closed in $U_{1}$ because $U_{1}$ is Hausdorff. Then

$$
\begin{aligned}
\mathrm{Cl}_{U_{1}}(Q \cap W) & \subset \mathrm{Cl}_{U_{1}}(V \cap Q \cap W) \cup\left(\left(\mathrm{Cl}_{U_{2}}(Q) \cap W\right)-V\right) \\
& \subset \bigcup_{k=1}^{r} \mathrm{Cl}_{U_{1}}\left(V_{z_{k}} \cap W\right) \cup W \subset T-\partial_{U_{1}}(W) . \cdot
\end{aligned}
$$

Denote by $W_{j}(j \in J)$ the connected components of $U_{1} \cap U_{2}$. We can take an open neighborhood $Q$ of $x_{2}$ in $U_{2}$ such that $\mathrm{Cl}_{U_{2}}(Q)$ is compact (because $U_{2}$ is Hausdorff and locally compact). 
(3.4) $x_{1} \in \mathrm{Cl}_{U_{1}}\left(\bigcup_{j \in J} \partial_{U_{1}}\left(Q \cap W_{j}\right)\right)$.

Pr o of. Suppose this property is false. Then, since $T$ is locally connected there exists a connected open neighborhood $V$ of $x_{1}$ in $U_{1}$ such that $V \cap$ $\partial_{U_{1}}\left(Q \cap W_{j}\right)=\emptyset$ for each $j \in J$.

By (3.1) we have

$$
x_{1} \in \mathrm{Cl}_{U}\left(U_{1} \cap Q\right)=\mathrm{Cl}_{U_{1}}\left(\bigcup_{j \in J}\left(Q \cap W_{j}\right)\right) .
$$

Thus there exists $j_{0} \in J$ such that $V \cap Q \cap W_{j_{0}} \neq \emptyset$. Moreover,

$$
\partial_{V}\left(V \cap Q \cap W_{j_{0}}\right) \subset V \cap \partial_{U_{1}}\left(Q \cap W_{j_{0}}\right)=\emptyset,
$$

so $V=V \cap Q \cap W_{j_{0}}$ because $V$ is connected. Therefore $x_{1} \in V \subset Q \cap W_{j_{0}} \subset$ $U_{1} \cap U_{2}$, yielding that $x_{2}$ is not in $S\left(x_{1}\right)$ because $U_{2}$ is Hausdorff, which is a contradiction.

Suppose first that $W_{j}$ satisfies the hypothesis of (3.3) for all $j \in J$.

(3.5) In this case $\partial_{U_{1}}\left(Q \cap W_{j}\right) \subset \partial_{U_{2}}(Q)$ for all $j \in J$.

Proof. For all $j \in J$, by (3.3) and since $W_{j}$ is open, we have

$$
\begin{aligned}
\partial_{U_{1}}\left(Q \cap W_{j}\right) & =\partial_{U_{1}}\left(Q \cap W_{j}\right) \cap W_{j}=\partial_{W_{j}}\left(Q \cap W_{j}\right) \\
& =\partial_{U_{2}}\left(Q \cap W_{j}\right) \cap W_{j} \subset \partial_{U_{2}}(Q) \cap W_{j} .
\end{aligned}
$$

By (3.4) and (3.5) we obtain

(3.6) $x_{1} \in \mathrm{Cl}_{T}\left(\partial_{U_{2}}(Q)\right)$ in this case.

Since $\partial_{U_{2}}(Q) \cap S\left(x_{1}\right) \subset\left(S\left(x_{1}\right)-\left\{x_{2}\right\}\right) \cap U_{2}=\emptyset$, for each $z \in \partial_{U_{2}}(Q)$ there exist open neighborhoods $V_{1}^{z}$ of $x_{1}$ and $V_{2}^{z}$ of $z$ such that $V_{1}^{z} \cap V_{2}^{z}=\emptyset$. Since $\partial_{U_{2}}(Q)$ is compact we have

$$
\partial_{U_{2}}(Q) \subset V_{2}^{z_{1}} \cup \ldots \cup V_{2}^{z_{r}}
$$

for some $z_{1}, \ldots, z_{r} \in \partial_{U_{2}}(Q)$. So $V=V_{1}^{z_{1}} \cap \ldots \cap V_{1}^{z_{r}}$ is an open neighborhood of $x_{1}$ such that $V \cap \partial_{U_{2}}(Q)=\emptyset$, which contradicts (3.6). Therefore there exists some connected component $P$ of $U_{1} \cap U_{2}$ which does not satisfy the hypothesis of (3.3), i.e. there exists $y_{0} \in \partial_{U_{2}}(P)$ such that for every neighborhood $V$ of $y_{0}$ in $U_{2}$ we have $\mathrm{Cl}_{U_{1}}(V \cap P) \cap \partial_{U_{1}}(P) \neq \emptyset$.

Take an open neighborhood $V$ of $y_{0}$ in $U_{2}$ such that $\mathrm{Cl}_{U_{2}}(V)$ is compact and take $y_{1} \in \mathrm{Cl}_{U_{1}}(V \cap P) \cap \partial_{U_{1}}(P)$. Suppose that for every $z \in \partial_{U_{2}}(V \cap P)$ $\cap \partial_{U_{2}}(P)$ there exist open neighborhoods $Q_{1}^{z}$ of $y_{1}$ and $Q_{2}^{z}$ of $z$ such that $Q_{1}^{z} \cap Q_{2}^{z} \cap P=\emptyset$. Since $\partial_{U_{2}}(V \cap P) \cap \partial_{U_{2}}(P)$ is compact (being closed in $\left.\mathrm{Cl}_{U_{2}}(V)\right)$, we obtain open sets $Q_{1}$ and $Q_{2}$ such that $y_{1} \in Q_{1}, \partial_{U_{2}}(V \cap P) \cap$ $\partial_{U_{2}}(P) \subset Q_{2}$, and $Q_{1} \cap Q_{2} \cap P=\emptyset$. Therefore

$$
\mathrm{Cl}_{U_{2}}(V \cap P)-Q_{2}=\left(\mathrm{Cl}_{U_{2}}(V \cap P) \cap P\right)-Q_{2} \subset P .
$$


Moreover, $\mathrm{Cl}_{U_{2}}(V \cap P)-Q_{2}$ is compact because it is closed in $\mathrm{Cl}_{U_{2}}(V)$, so it is closed in $U_{1}$ because $U_{1}$ is Hausdorff. Thus

$$
\begin{aligned}
y_{1} & \in \mathrm{Cl}_{U_{1}}(V \cap P) \cap \partial_{U_{1}}(P) \\
& \subset \mathrm{Cl}_{U_{1}}\left(\left(Q_{2} \cap P\right) \cup\left(\mathrm{Cl}_{U_{2}}(V \cap P)-Q_{2}\right)\right) \cap \partial_{U_{1}}(P) \\
& =\left(\mathrm{Cl}_{U_{1}}\left(Q_{2} \cap P\right) \cup\left(\mathrm{Cl}_{U_{2}}(V \cap P)-Q_{2}\right)\right) \cap \partial_{U_{1}}(P) \\
& \subset \mathrm{Cl}_{U_{1}}\left(Q_{2} \cap P\right) \cap \partial_{U_{1}}(P),
\end{aligned}
$$

implying $Q_{1} \cap Q_{2} \cap P \neq \emptyset$, which is another contradiction. Therefore there exists $y_{2} \in \partial_{U_{2}}(V \cap P) \cap \partial_{U_{2}}(P)$ such that $P \cap Q_{1} \cap Q_{2} \neq \emptyset$ for all neighborhoods $Q_{1}$ and $Q_{2}$ of $y_{1}$ and $y_{2}$ respectively. Moreover, $y_{2} \in S\left(y_{1}\right)$ because $y_{2} \neq y_{1}$ by (3.2). So the proof of (2.2) is finished when (2.2)(i) is satisfied.

The proof of (2.2) in the other case is an easy exercise.

4. Examples. More examples of the type we shall consider can be found in $[\mathrm{HR}]$.

(4.1) Consider two copies of $\mathbb{R}$ and identify each point of the open interval $(0,1)$ in the first copy with the corresponding point in the second copy. The quotient space is a manifold satisfying $(\mathrm{A})$ and $(\mathrm{B})$.

(4.2) Consider an infinite family $F$ of disjoint non-empty open subsets of $\mathbb{R}$, and let $U$ be their union. We can take $F$ such that any neighborhood of each point of $\mathbb{R}-U$ contains infinite sets of $F$. Then, taking two copies of $\mathbb{R}$ and identifying each point of $U$ in the first copy with the corresponding point in the second one, we obtain a quotient space which is a manifold satisfying (A) but not (B).

(4.3) Let $A, B$ and $C$ be non-collinear points in $\mathbb{R}^{2}$, let $\Delta$ be the domain bounded by the triangle with vertices those points, and let $h$ be the distance between $A$ and the straight line $r$ which contains $B$ and $C$. For $0<\varepsilon<h$ let $r_{\varepsilon}$ be the straight line which is parallel to $r$ and at a distance of $\varepsilon$ and $h-\varepsilon$ to $r$ and $A$ respectively. With this notation we have the homeomorphism $\varphi: \Delta \rightarrow \Delta$ which carries, by radial projection with center $A$, each $r_{\varepsilon} \cap \Delta$ to $r_{h-\varepsilon} \cap \Delta$. Then, taking two copies of $\mathbb{R}^{2}$ and identifying each point $(x, y) \in \Delta$ in the first copy with the point $\varphi(x, y)$ in the second one, we get a quotient space which is a manifold satisfying (B) but not (A).

(4.4) For each positive integer $n$ let $\Delta_{n}$ be the domain in $\mathbb{R}^{2}$ bounded by the triangle with vertices $A_{n}=\left(m_{n}, n\right), B_{n}=(1 /(n+1), 0)$ and $C_{n}=$ $(1 / n, 0)$, where $m_{n}=(2 n+1) /(2 n(n+1))$. For each $n$ and $0<\varepsilon<n$ let $r_{n, \varepsilon}$ be the straight line containing $B_{n}$ and $\left(m_{n}, \varepsilon\right)$, and let $s_{n, \varepsilon}$ be the straight line containing $C_{n}$ and $\left(m_{n}, \varepsilon\right)$. Defining $\Delta_{n, 1}=\left\{(x, y) \in \Delta_{n}\right.$ : $\left.x \leq m_{n}\right\}$ and $\Delta_{n, 2}=\left\{(x, y) \in \Delta_{n}: x \geq m_{n}\right\}$, we have the homeomorphism 
$\varphi_{n}: \Delta_{n} \rightarrow \Delta_{n}$ which carries, by radial projection with center $A_{n}$, each $r_{n, \varepsilon} \cap \Delta_{n, 1}$ to $r_{n, n-\varepsilon} \cap \Delta_{n, 1}$, and each $s_{n, \varepsilon} \cap \Delta_{n, 2}$ to $s_{n, n-\varepsilon} \cap \Delta_{n, 2}$.

Consider two copies of $\left\{(x, y) \in \mathbb{R}^{2}: y>0\right\}$ and, for each $n$, identify $(x, y) \in \Delta_{n}$ in the first copy with $\varphi_{n}(x, y)$ in the second one. Then the quotient space $T$ is a manifold which satisfies neither (A) nor (B). So $T$ does not satisfy the hypotheses of (2.2), and it is easy to check that $T$ does not satisfy the conclusion of that lemma either.

5. Application to foliations. A foliation $\mathcal{F}$ on a manifold $X$ can be defined by an open covering $\left\{U_{i}\right\}_{i \in I}$ of $X$ and surjective submersions $f_{i}$ of $U_{i}$ on manifolds $T_{i}$ with connected fibers, such that there exist homeomorphisms $h_{i j}: f_{j}\left(U_{i} \cap U_{j}\right) \rightarrow f_{i}\left(U_{i} \cap U_{j}\right)$ so that $f_{i}=h_{i j} f_{j}$ on $U_{i} \cap U_{j}$. If $T$ is the disjoint union of the $T_{i}$ 's, a representative of the holonomy pseudogroup of $\mathcal{F}$ is the pseudogroup $\mathcal{H}$ generated by the local homeomorphisms $h_{i j}$ of $T$. Another choice of $\left(U_{i}, f_{i}, h_{i j}\right)$ would lead to a pseudogroup equivalent to $\mathcal{H}[\mathrm{H} 2]$.

From [W] we know that any representative of the holonomy pseudogroup acting on a Hausdorff manifold is quasi-analytical if and only if the graph of $\mathcal{F}$ is Hausdorff. For instance, this is the case for Riemannian or transversely analytical foliations.

$\mathcal{F}$ is said to be developable if there exists a covering mapping $\pi: \widetilde{X} \rightarrow X$ such that the space $\widetilde{T}$ of leaves of $\pi^{*} \mathcal{F}$ is a manifold [H2]. (Such foliations are characterized in [H3].) In this case the covering transformations preserve $\pi^{*} \mathcal{F}$, thus Aut $(\pi)$ acts on $\widetilde{T}$ and it is easy to check that the pseudogroup $\widetilde{\mathcal{H}}$ generated by this action is a representative of the holonomy pseudogroup of $\mathcal{F}$.

We shall say that $\mathcal{F}$ is induced by a triple $(\pi, D, h)$ if $\pi: \widetilde{X} \rightarrow X$ is a covering mapping, $D$ is a submersion of $\widetilde{X}$ onto a Hausdorff manifold $T$ and $h: \operatorname{Aut}(\pi) \rightarrow \operatorname{Homeo}(T)$ is an injective homomorphism such that $\pi^{*} \mathcal{F}$ is induced by $D$ (its leaves are the connected components of the fibers of $D$ ) and $D$ is $h$-equivariant $(D \gamma=h(\gamma) D$ for every deck transformation $\gamma)$.

In this case it is easy to check that the space $\widetilde{T}$ of leaves of $\pi^{*} \mathcal{F}$ is a manifold, which in general is not Hausdorff, so $\mathcal{F}$ is a developable foliation. Then the pseudogroup $\widetilde{\mathcal{H}}$ generated by the action of $\operatorname{Aut}(\pi)$ on $\widetilde{T}$ is a representative of the holonomy pseudogroup of $\mathcal{F}$. We also have the pseudogroup $\mathcal{H}$ generated by the image $H$ of $h$. This leads to the question whether $\mathcal{H}$ is equivalent to $\tilde{\mathcal{H}}$. Using (1.1), (2.1) and (2.3) it is easy to prove the following result which gives a negative answer in some cases.

(5.1) Proposition. Assume that, in the above situation, the graph of $\mathcal{F}$ is Hausdorff. Then:

(i) If $\widetilde{T}$ is not Hausdorff then $\mathcal{H}$ is not equivalent to $\widetilde{\mathcal{H}}$. 
(ii) If $\pi^{\prime}: \widetilde{X}^{\prime} \rightarrow X$ is another covering mapping such that the space $\widetilde{T}^{\prime}$ of leaves of $\pi^{*} \mathcal{F}$ is a Hausdorff manifold, then $\widetilde{T}$ is Hausdorff.

We have a simple example by taking on $X=\mathbb{R}^{2}-\{(0,0)\}$ the foliation $\mathcal{F}$ given by the triple $(\pi, D, h)$ where $\pi$ is the trivial covering mapping, $D$ is the projection of $X$ onto the first axis, and $h$ is the trivial homomorphism. In this case we conclude that $\widetilde{\mathcal{H}}$ is not equivalent to $\mathcal{H}$, and the space of leaves of any lifting of $\mathcal{F}$ to any covering of $X$ is not Hausdorff.

Let $\mathcal{F}$ be a foliation on a manifold $X$ given by a transverse $(G, T)$ structure [H1], i.e. $G$ is a group of homeomorphisms of a manifold $T$ and $\mathcal{F}$ is defined by a cocycle $\left(U_{i}, f_{i}, g_{i j}\right)$ with $f_{i}: U_{i} \rightarrow T$ and $g_{i j} \in G$. In this case, if $G$ acts quasi-analytically it can be proved (Ehresmann) that $\mathcal{F}$ is given by a triple $(\pi, D, h)$ as above where $H \subset G$. Moreover, if $X$ is compact, $T$ a Riemannian manifold and $G$ a group of isometries, then $\widetilde{T} \equiv T$ canonically (thus $\widetilde{\mathcal{H}} \equiv \mathcal{H}$ ). For instance, this is the case for Lie foliations on compact manifolds $[\mathrm{M}]$.

\section{References}

[H1] A. Haefliger, Groupodes d'holonomie et classifiants, in: Structures Transverses des Feuilletages, Toulouse 1982, Astérisque 116 (1984), 70-97.

[H2] -, Pseudogroups of local isometries, in: Differential Geometry, Res. Notes in Math. 131, Pitman, 1985, 174-197.

[H3] - , Leaf closures in Riemannian foliations, in: A Fête of Topology, Academic Press, Boston, Mass., 1988, 3-32.

[HR] A. Haefliger et G. Reeb, Varits (non sparées) à une dimension et structures feuilletes du plan, Enseign. Math. 3 (1957), 107-125.

[M] P. Molino, Gomtrie globale des feuilletages riemanniens, Nederl. Akad. Wetensch. Indag. Math. 44 (1982), 45-76.

[W] H. E. Winkelnkemper, The graph of a foliation, Ann. Global Anal. Geom. 1 (3) (1983), 51-75.

UNIVERSIDADE DE SANTIAGO DE COMPOSTELA DEPARTAMENTO DE XEOMETRÍA E TOPOLOXÍA COLEXIO UNIVERSITARIO DE LUGO 27071 LUGO, SPAIN

E-MAIL: XTZZL002@SEINL.LUGO.USC.ES 